\title{
BRAILLE GRAPH PAPER IN CHARTING of
} STRENGTH/DURATION CURVES

(Reprinted by kind permission of "Physiotherapy" and Miss Joyce Massey, the author).

\author{
By JOYCE MASSEY, M.C.S.P. (Teacher's Certificates)
}

School of Physiotherapy, Royal National Institute for the Blind, 204, Great Portland Street, London, W.1.

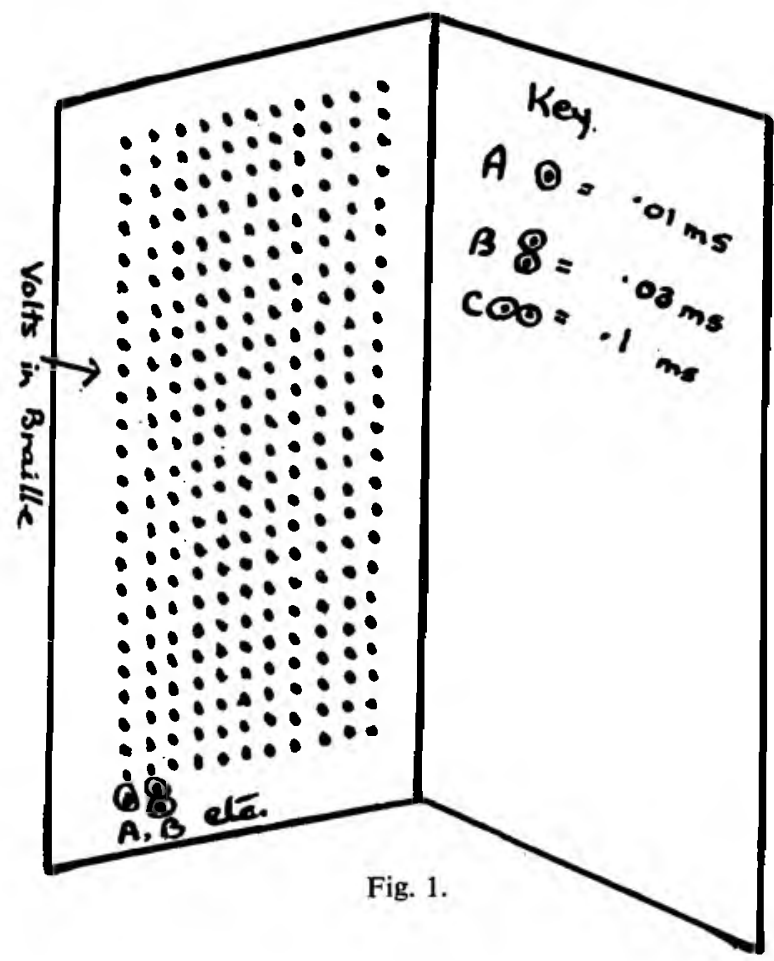

At the Royal National Institute for the Blind we have endeavoured for some time to design a suitable form of braille graph paper on which a blind physiotherapist can produce an accurate chart of the strength/duration muscle test on a par with that used by a sighted person. The design of this paper has now been perfected and this article is written with the hope that Superintendent Physiotherapists and others may learn of what the blind are capable in this field.

A large double foolscap sheet of special paper is brailled on the inside left-hand page with the pulse durations marked along the base in letters. The usual 10 pulses of $300 \mathrm{~m} . \mathrm{s}$., 100 m.s., 30 m.s., 10 m.s., 3 m.s., 1 m.s., .3 m.s., 1 m.s., $.03 \mathrm{~m} . \mathrm{s}$. and $.01 \mathrm{~m} . \mathrm{s}$. are represented by the letters of the braille alphabet from A-J. For example, $J$ is used for the longest $300 \mathrm{~m} . \mathrm{s}$. pulse, I for the $100 \mathrm{~m}$.s. pulse, and so on, with A representing the brief $.01 \mathrm{~m} . \mathrm{s}$. pulse. A key in braille appears on the right-hand inner page.

The placing of the pulses on this braille side of the paper is the same as on sighted graph paper, e.g. the $300 \mathrm{~m}$.s. pulse $(J)$ is placed on the right of the base, and the $.01 \mathrm{~m}$.s. pulse $A$ on the extreme left.

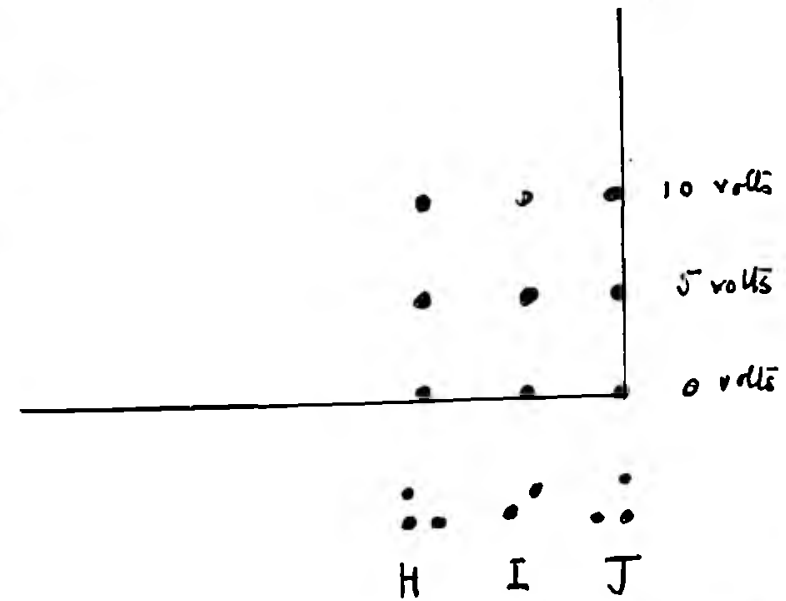

Fig. 1a.

$$
(30 \mathrm{~ms})(100 \mathrm{~ms})(300 \mathrm{~ms})
$$

Fig. 1. Braille side of paper (reduced in size). Actual size of each page 13 in. by 10 in.

Fig. 1a. Section of braille side (showing actual distance between braille dots).

Immediately above each of these pulses is a file or line of braille dots. These represent the vertical lines of the graph paper.

The strength of the voltage is marked from $0-120$ volts in numerals proceeding in 5 volts on both the left and right sides of this page. Exactly opposite each of the 5 volt markings is a row of transverse dots which stretch across the page and represent the abscissae or transverse lines of the graph paper.

To assist the blind physiotherapist who is using the paper for the first time, these markings are explained by a further key on the right-hand page. (Fig. 1 and 1a.)

If the paper is turned over to the reverse side, the rows of vertical and transverse dots are not felt. This is therefore known as the 'plain' side of the paper. However, on this side of the paper the pulse durations are also marked along the base and the voltage values up the left-hand side, with the difference that the pulse durations now read from left to right with $300 \mathrm{~m} . \mathrm{s}$. (J) on the left and the .01 pulse (A) on the extreme right of the base. (Fig, 2.)

The voltage strength, as before stated, is only marked on the left-hand side; both these markings could be omitted, 


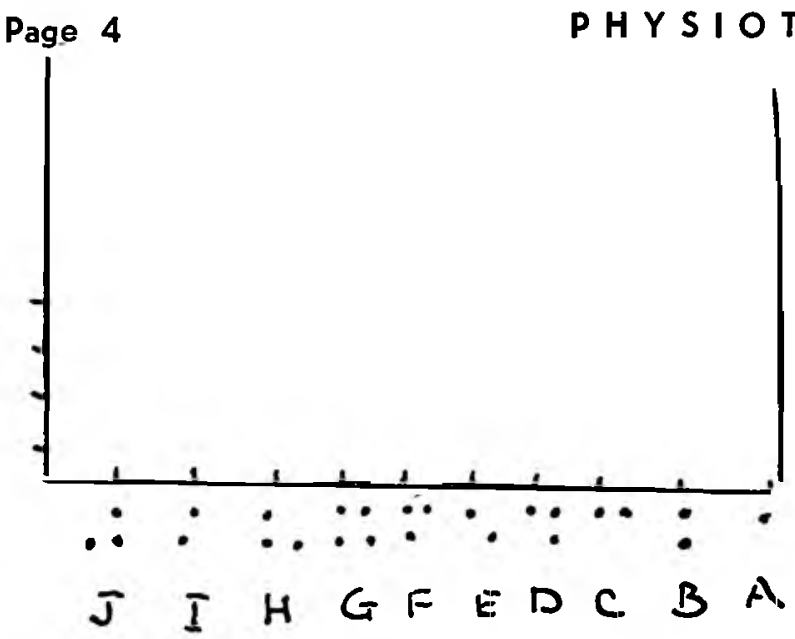

Fig. 2. Plain side (showing reversal in position of pulse markings).

but are useful if a check of pulse and voltage is required on this page.

Before the blind physiotherapist can draw the graph he needs the following accessories:

1. A braille point (this is a small spike fitted with a wooden handle).

2. A spur wheel (various types of these are available, but they are all based on the principle of a small wheel with a sharply spurred circumference, which is capable of rotation when it is pushed along. It is also fitted with a handle.)

3. A hard rubber mat (approximately $10 \mathrm{in.} \mathrm{by} 8$ in.)

4. A piece of foam rubber (about the same size).

5. A sheet of carbon paper.

6. Some metallized adhesive tape (the adhesive backing of this may be stuck on to the graph paper and such details as date, type of machine used, name of patient, name of muscle, etc., may be brailled on the metal side).

\section{Use of Paper}

The hard rubber mat with the foam rubber on top is placed on the table. The braille graph paper is then opened out, with the braille side uppermost, facing the operator. The charting may be done without the foam rubber if desired (simply placing the paper on the hard rubber mat), but as holes have to be pricked in the braille paper with the braille point, these have been found to be better if the foam rubber is used addititionally.

The physiotherapist then holds the braille spike in his right hand and selects the given pulse duration with his fingers and follows up the vertical line of braille dots until the appropriate voltage is reached for this pulse.

To record this voltage the physiotherapist then pricks a mark into the graph paper on the exact point required (Fig. 3). It is essential for the physiotherapist to be, of course, precise and consistent in the positioning of this mark, and great care is taken to see that it is either on the appropriate dot or on an exact line with it for the in between voltages.'

The voltage needed for each of the succeeding pulses is then pricked through the paper in this way.

When this preliminary charting is completed the foam rubber is removed. The graph paper is then turned over to the plain side and a carbon sheet is placed beneath it, on top of the hard rubber mat. The blind operator has to recognize

the carbon side of the carbon paper by feel. This provides a slightly sticky feel which is quite different from the smoothness of the opposite side and presents no difficulty.

The dots which have been pricked through can now be felt on this, originally plain, side of the paper. These form the skeleton of the graph.

The spur wheel is now taken and the circumference of the wheel rolled from dot to dot, thus joining them up. (Fig. 4).

The paper is then reversed again back to the braille side, where a feelable and visual graph is present.

If the muscle test is performed by the medical officer with a blind physiotherapist assisting, or by two physiotherapists (one or both of whom could be blind), then the charting can be done at the same time as the test.

In the rather less likely event of the blind physiotherapist being left on his own to do both test and chart, the latter is done afterwards, a note of the voltage required for given pulses having been taken and used in later charting.

The performance of the charting may sound a little formidable, but experience shows that it can be accurately and easily done.

A description of the exact way in which the blind physiotherapist does the test is outside the scope of this article, and is indeed so similar to the way of a sighted operator as not to warrant description.

However, it may be useful to know that if the test is done with the apparatus known as the 10-pulse constant voltage stimulator, only very slight alterations to this apparatus are required, namely, an additional resistance and jack socket. The latter is for the inclusion of the jack plug of a special audible voltmeter.

The cost of the alterations to the apparatus are very reasonable, being less than $\mathrm{R} 2$, whilst the audible meter would be part of the blind physiotherapists' own equipment. The cost of the graph paper is negligible.
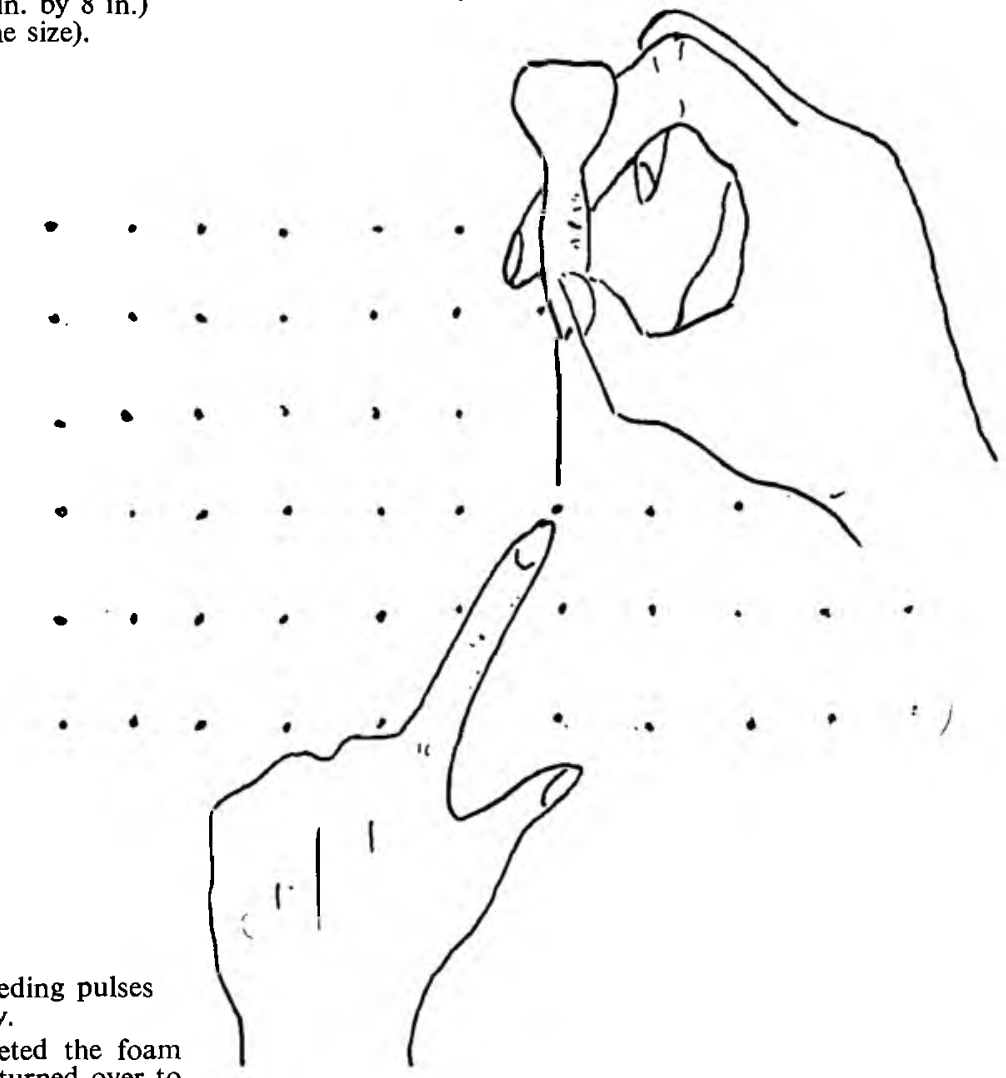

Fig. 3. To show use of braille point on the braille side of the paper. 


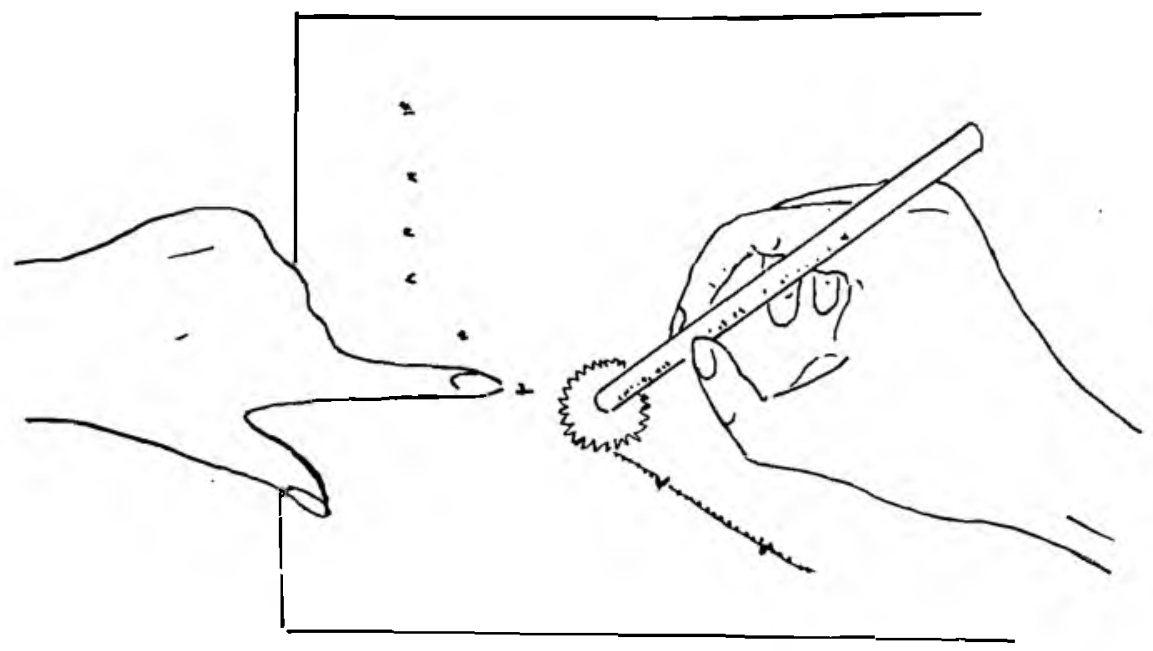

Fig. 4. To show use of spur wheel in joining up marks on 'plain' side.

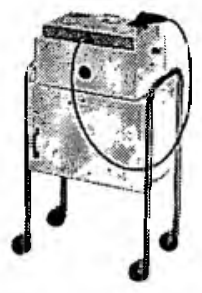

\section{THE BIRTCHER MEGASON VI ULTRASONIC UNIT}

To the more than 20,000 physicians now using ultrasonic therapy in the treatment of a host of acute and chronic conditions, this precision instrument adds new dimensions of accuracy and treatment ease.

Descriptives and medical journal reprints on request.
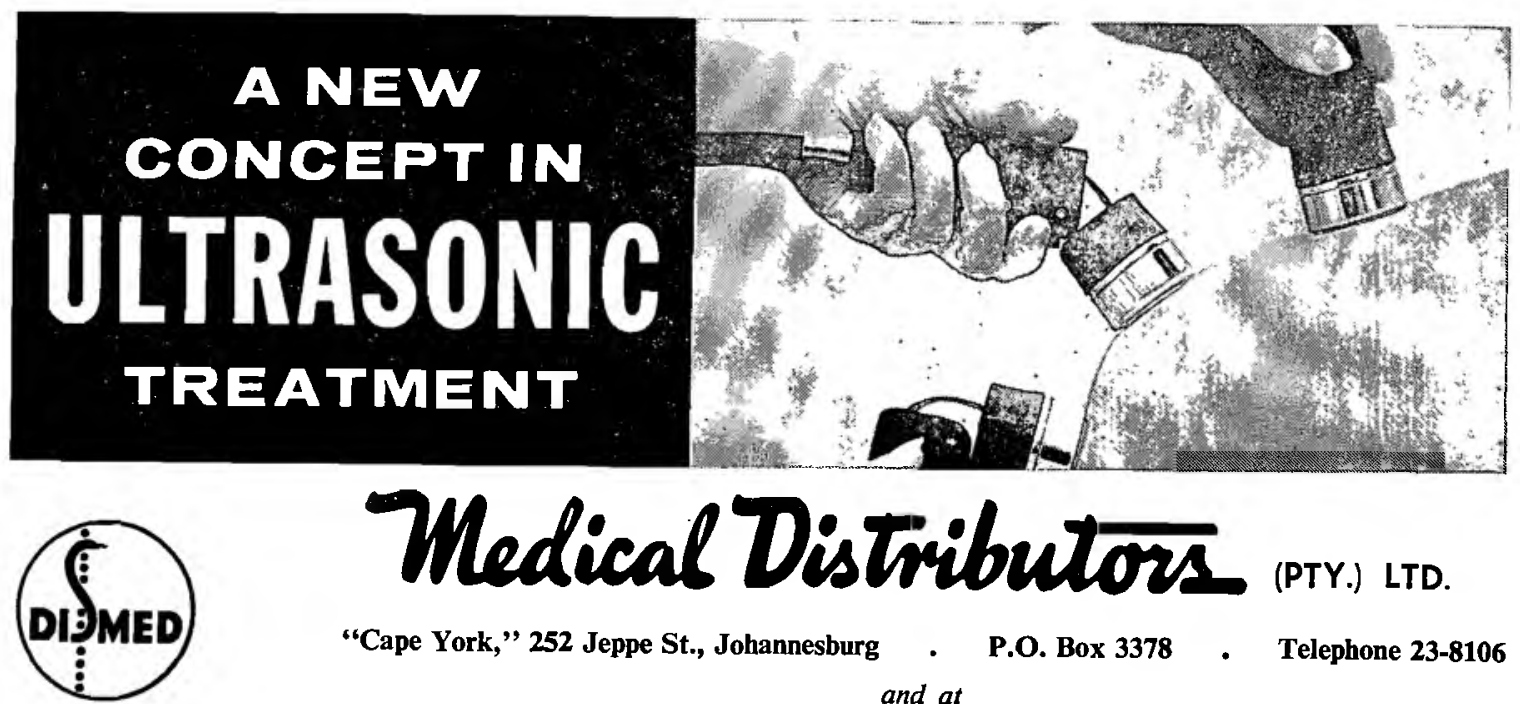

(PTY.) LTD.

“Cape York,” 252 Jeppe St., Johannesburg

P.O. Box 3378

Telephone 23-8106

and at

President House. 20 Barrack Street, Cape Town. P.O. Box 195. Telephone 41-1172 